\author{
SANDIA REPORT \\ SAND2008-5887 \\ Unlimited Release \\ Printed September 2008
}

\title{
Considerations for Developing Models of Multiphase Flow in Deformable Porous Media
}

Mario J. Martinez and Charles M. Stone

Prepared by

Sandia National Laboratories

Albuquerque, New Mexico 87185 and Livermore, California 94550

Sandia is a multiprogram laboratory operated by Sandia Corporation, a Lockheed Martin Company, for the United States Department of Energy's

National Nuclear Security Administration under Contract DE-AC04-94AL85000.

Approved for public release; further dissemination unlimited. 
Issued by Sandia National Laboratories, operated for the United States Department of Energy by Sandia Corporation.

NOTICE: This report was prepared as an account of work sponsored by an agency of the United States Government. Neither the United States Government, nor any agency thereof, nor any of their employees, nor any of their contractors, subcontractors, or their employees, make any warranty, express or implied, or assume any legal liability or responsibility for the accuracy, completeness, or usefulness of any information, apparatus, product, or process disclosed, or represent that its use would not infringe privately owned rights. Reference herein to any specific commercial product, process, or service by trade name, trademark, manufacturer, or otherwise, does not necessarily constitute or imply its endorsement, recommendation, or favoring by the United States Government, any agency thereof, or any of their contractors or subcontractors. The views and opinions expressed herein do not necessarily state or reflect those of the United States Government, any agency thereof, or any of their contractors.

Printed in the United States of America. This report has been reproduced directly from the best available copy.

Available to DOE and DOE contractors from

U.S. Department of Energy

Office of Scientific and Technical Information

P.O. Box 62

Oak Ridge, TN 37831

Telephone: $\quad$ (865) 576-8401

Facsimile: $\quad$ (865) 576-5728

E-Mail:_reports@adonis.osti.gov

Online ordering: http://www.osti.gov/bridge

Available to the public from

U.S. Department of Commerce

National Technical Information Service

5285 Port Royal Rd.

Springfield, VA 22161

Telephone:

(800) 553-6847

Facsimile:

(703) 605-6900

E-Mail:

orders@ntis.fedworld.gov

Online order:

http://www.ntis.gov/help/ordermethods.asp?loc=7-4-0\#online

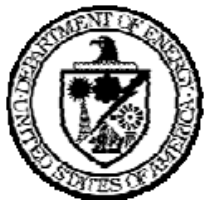


SAND2008-5887

Unlimited Release

Printed September 2008

\title{
Considerations for Developing Models of Multiphase Flow in Deformable Porous Media
}

\author{
Mario J. Martinez \\ Thermal and Fluid Processes Department 1514 \\ Charles M. Stone \\ Strategic Initiatives Department 1525 \\ Sandia National Laboratories \\ P.O. Box 5800 \\ Albuquerque, New Mexico 87185-MS0836
}

\begin{abstract}
This document summarizes research and planning for the development of a numerical simulation capability for nonisothermal multiphase, multicomponent transport in heterogeneous deformable porous materials. Particular attention is given to describing a mathematical formulation for flow in deformable media and for numerical techniques for dealing with phase transitions. A development plan is formulated to provide a computational capability motivated by current and future needs in geosystems management for energy security.
\end{abstract}




\section{ACKNOWLEDGMENTS}

We appreciate productive discussions with Prof. MF Wheeler from the Center for Subsurface Modeling at the University of Texas at Austin concerning computational multiphase flow. We also thank Dr. Rick Dean, Chevron, for discussions on phase transitions and other aspect of multiphase flow modeling. 


\section{CONTENTS}

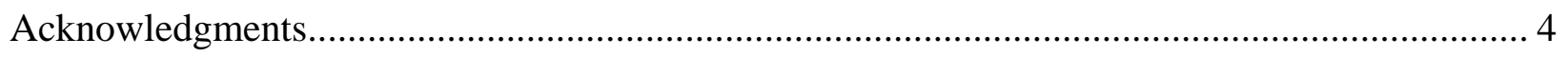

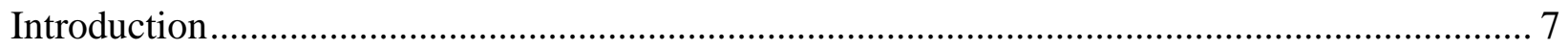

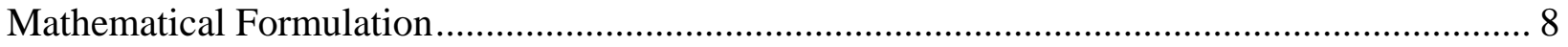

Literature on Deformable Porous Media ……………………............................................. 8

Nonisothermal Multiphase Flow in Deformable Porous Media............................................ 11

Balance Equations for a Phase ................................................................................ 11

Balance Equation for Mass Components ...................................................................... 13

Heat Transport .................................................................................................. 13

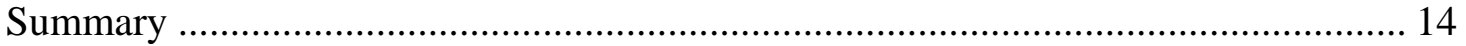

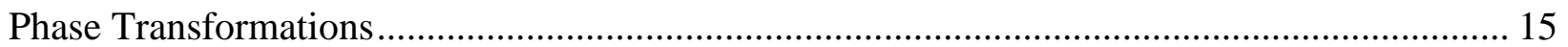

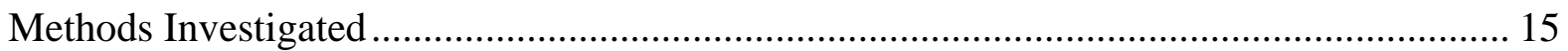

Relaxed Volume Balance........................................................................................ 15

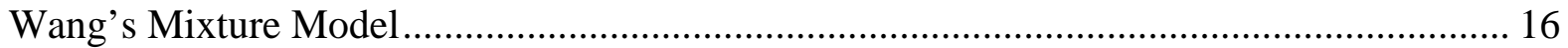

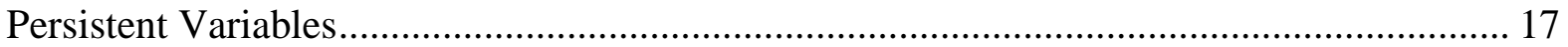

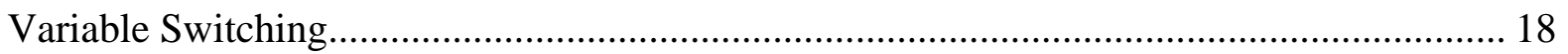

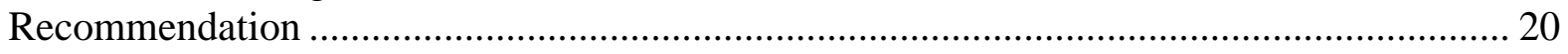

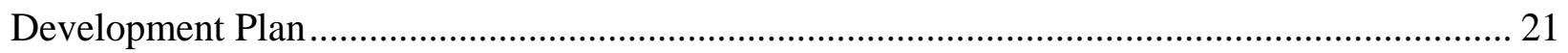

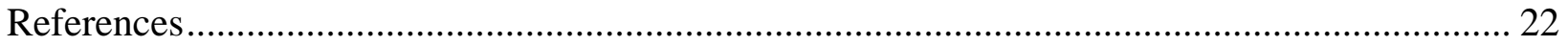




\section{INTRODUCTION}

The development of energy systems and resources requires the understanding and predictive simulation of complex processes in earth systems. For example, the development and licensing of nuclear waste repositories is a key path to the expansion of nuclear power. This path is becoming more dependent on modeling and simulation coupling processes such geomechanical response, thermal, fluid flow and chemical response of the natural and engineered systems. The US has large reserves of coal, approximately 200 years at current use levels. As long as this resource can be used cleanly, these reserves provide a backbone of energy security. Carbon reuse and sequestration may provide a path to clean utilization. Similar areas for model utilization are oil and gas reservoirs, advanced recovery from tar sands and shales, underground storage of hydrogen, natural gas and oil, and aquifers for new water resources. Any significant progress in these areas will require new coupled modeling and simulation approaches. It is important to note that very few parallel processing commercial and/or research software tools exist for simulating complex processes such as coupled multiphase flow with chemical transport and geomechanics. Current computational limitations place significant restrictions on realistic problems that can be solved.

Predictive computational simulation of the coupled-physics associated with geosystems is a critical enabling technology for their optimal management. A major stumbling block to highfidelity modeling and simulation of geosystems is the lack of viable, robust, and efficient computational technologies for describing multiphase, multicomponent chemically reactive fluid mixtures in heterogeneous geologic media. In compositional descriptions, the fluid mixtures partition into various phases, depending on the local thermodynamic state. Flow and transport processes under temperature, pressure and compositional gradients result in phases disappearing and/or appearing, a highly complex and difficult phenomena to capture in simulations. However, the accuracy, and indeed usability, of modeling and simulation for geosystems hinges on proper accounting for this dynamic reactive phase behavior.

The objective of this LDRD project (project no. 125883) was to investigate computational methods and form a development plan for computer models of multiphase, multicomponent deformable geosystems. An intended benefit of this work is that it forms the foundation to enable Sandia to develop comprehensive computational tools for developing and managing the nation's energy and natural resources. As a collateral benefit, this project performs research supporting the growing partnership between Sandia National Laboratories and the University of Texas at Austin (UT) in the geosciences.

In the following, we first describe the mathematical formulation describing nonisothermal, multiphase, multicomponent flow in deformable porous materials. This forms the basic framework for the geosystems models to be ultimately developed in future work. This is followed by a literature review on techniques in current use for describing phase transitions in multiphase, multicomponent systems, including recommendations for a successful path to efficient computational thermodynamics for these systems. Finally, a plan for the future development of these models is suggested. 


\section{MATHEMATICAL FORMULATION}

\section{Literature on Deformable Porous Media}

The following is not a comprehensive literature review of treatments of multiphase flow and mechanics. We review some selected papers which are highly relevant to the objectives of this project.

De Boer (1998) presents a good review and history of the theory of porous media in general, including the historical development of theory for deformable porous media. Treatments of coupled deformation and flow in porous materials begin with the works of von Terzaghi (1943) and Biot (1941) on consolidation. These papers introduce the important concept of effective stress in saturated porous media for describing the "settlements of soils under load." In a more recent paper Schrefler \& Scotta (2001) define the mixture fluid pressure for an air and water system as $p=S_{w} p_{w}+S_{a} p_{a}$, which is used in the effective stress principle, augmented by the volumetric deformation of solid particles (Biot \& Willis, 1957),

$$
\sigma_{i j}^{e f f}=\sigma_{i j}+\alpha \delta_{i j} p
$$

to couple the fluid pressures with the deformation mechanics. Generalized two-phase flow equations govern the motion of fluids. Their formulation includes the rate of change of grain volume due to strain rates and average pressure changes; these terms couple the fluid flow to the deformation. The book by Lewis \& Schrefler, (1998) discusses the formulation in more detail. Owing to the dynamic deformation of the porous skeleton, the formulation is naturally in terms of a moving (accelerating) coordinate system. This gives rise, for example, to accelerations relative to the gravitational term in the Darcy equations, and to a dilatation term in the fluid mass balance equations. Other formulations, to be discussed next, include an evolution equation for porosity changes. Also notable for this project is that they do not treat the phase appearance/disappearance problem directly, instead they allow trace amounts of gas to exist (numerically) under liquid saturated conditions.

In general, the linear momentum balance for the mixture is simplified to the form (Schrefler \& Scotta, 2001),

$$
\sigma_{k l, k}+\rho b_{l}=\rho \ddot{u}_{l}+\varsigma \dot{u}_{l}
$$

where $\rho=(1-\phi) \rho_{s}+\phi S_{w} \rho_{w}+\phi S_{a} \rho_{a}$ (there appears to be a typographical error in their eqn. (12)) is the mixture density, $\varsigma$ is a damping coefficient, and $u_{l}$ is the displacement vector. Other improvements to the theory of Biot (which Schrefler \& Scotta apply in their formulation) include the dependence on porosity change of the macroscopic strain energy, see Lo et al., 2002. This leads to an evolution equation for porosity, which Lo et al. show can be further distilled into an equation in terms of fluid and solid dilatations, as proposed originally by Biot. This new development also includes the effects of pore volume strain (porosity changes). Schrefler \& Scotta (2001) go on to discuss the limiting forms of their dynamical formulation for quasi-statics and statics (with respect to the solid mechanics).

Numerically, coupling solid mechanics to fluid flow often introduces disparate time and length scales. In geologic applications (oil \& gas exploitation, for example), the fluid flow and solid 
mechanics are often decoupled numerically and solved sequentially with lagged updating of data (porosity and pressures). Dean et al., (2003) take the viewpoint of deformable porous media as a problem in coupling between existing codes for solid mechanics and flow in porous media. The flow model is coupled to the deformation through its effect on porosity, and therefore permeability, and the effect of strain rate. The solid mechanics problem is coupled to the flow through the fluid pressure which enters into the effective stress via a Biot-type of model, similar to Eq. (1). Dean et al. (2003) compared the full spectrum of coupling techniques for geomechanics and flow in porous media. They investigated (a) explicit coupling, with flow calculations performed at every step and displacement computed at selected time steps, (b) iterative coupling, where flow and geomechanics are computed sequentially within a time step, and (c) fully coupled, where the nonlinear equations for flow and mechanics are solved simultaneously. All three methods were implemented in the same code (ACRES, Arco's comprehensive reservoir simulator), in an attempt to provide a closer comparison of the methods and diminish differences in their numerical implementations. In their applications they ignore the dependence of permeability on variations in porosity (hence deformation).

As noted by Dean et al., the primary attraction of the explicit coupling is that the disparate timescales in flow vs. geomechanics can be handled efficiently. In their applications, the flow timescale was shorter than the mechanics, i.e., they advanced the flow for several time steps before taking a mechanics time step. They discuss scaling arguments to give a criterion for estimating the mechanics time step compared to the flow time step. They also discuss a method of estimating an effective compressibility used only to enhance numerical convergence but not as part of the residual equations.

The primary attraction of the iteratively coupled approach is that it enables a quick construction of coupled geomechanics and flow using existing code. The drawback is that the segregated approach to solving the systems will reduce the convergence rate to linear (from quadratic, say, if using Newton on the coupled system). The coupled approach probably converges faster but may be inefficient on problems with disparate timescales. They compare the various techniques on four different problems, three of which can be modeled using matrix compressibility without need of coupled mechanics. One problem involved a producing region inside of a non-producing impermeable region. This one displays effects that could only be modeled with the coupled physics, and for which the fully coupled approach was the most efficient. The explicit method performed poorly on this problem. The other three problems show a slight advantage for the explicit scheme. Although this work considered only a few specific types of problems, the conclusion would seem to be that the iteratively coupled approach remained competitive on all problems, and requires a modest effort to program using existing codes, and thus seems like a good general technique.

The paper by Rutqvist et al (2001) does a good job of presenting a well-written general derivation of the governing equations for multiphase flow in deformable porous materials. The paper also compares four computer codes applicable to modeling thermohydromechanical phenomena in a general setting, though the work is aimed at modeling nuclear waste repository performance assessment in fractured media. The paper presents the derivation of a general balance equation which forms the basis for deriving multiphase mass, energy and linear momentum (mechanics) equations. The formulation includes a general energy balance, written 
so that evaporation/condensation can be modeled assuming equilibrium thermodynamics. These equations are written with respect to the motion of the solid skeleton, and hence introduce new terms arising from consideration of a deformable porous material. The linear momentum equation is written in terms of the total system stress and the effective stress concept can be introduced as a constitutive model. In addition, to accommodate plastic behavior, the effective stress is written in incremental form, such that thermal strain, grain compression and swelling of the porous material can be modeled.

The paper goes on to compare in detail the formulations of four available computer models for this problem type; see Tables 2-8 for a term-by-term comparison of the governing equations. All of the codes employ a modified effective stress law and a moisture-swelling strain model that depends on liquid saturation. The paper notes that the general mechanical behavior of unsaturated soil should depend on two independent stress variables, and therefore the general validity of the effective stress law cannot be assumed. Also, the swelling should depend on chemical composition. Improved constitutive formulations are discussed in the state surface approach presented by Alonso et al. (1990).

In a related paper, Rutqvist et al. (2002) discusses coupled thermal-hydrologic-mechanical (THM) modeling by coupling two existing codes, TOUGH2 and FLAC3D. TOUGH2 is a thermo-hydrologic code while FLAC3D can model thermo-hydrologic-mechanics problems, but is restricted to single phase fluid flow. According to the paper, FLAC3D solves the solid dynamics problem, including large strain by correcting the stress tensor for rotational strains. It also includes the effective stress formulation with a Biot effective stress parameter, $\alpha$ :

$$
\boldsymbol{\sigma}^{\prime}=\boldsymbol{\sigma}+\mathbf{I} \alpha P \text {. }
$$

Thus, in coupling the codes, the TOUGH2 simulation provides updated effective pressure and temperature for use in FLAC3D in the calculation of effective stress. A mechanics solution step provides updated stress, deformation and porosity. Updated permeability and capillary pressure follow from constitutive models. Thus, FLAC3D can be used without code modification, while TOUGH2 was only slightly modified by including a volumetric strain term, which can be added in as a volumetric source term. The codes were coupled via a separate batch program that controls the coupling and execution of the linked codes. Also needed are various interpolation routines, because the codes use different discretization schemes, and coupling functions which take in the requisite data from one code and process it as needed to compute the output for the other code. For example one coupling function would receive updated stress and strain, and use it to compute the corresponding updates in permeability, porosity and capillary pressure. It was noted that the coupling can be performed explicitly, wherein each code is executed in sequence, with data transfers occurring only at the beginning of each step, or implicitly, in which data transfers occur during each nonlinear iteration. The coupled codes were applied to two example problems, both using the explicit coupling approach. No information was given about the convergence properties of the simulations. It is noted that the sequentially coupled approach will work best when the hydromechanical changes are slow compared to the flow, while an implicit coupling may be necessary for higher strain rate problems. 


\section{Nonisothermal Multiphase Flow in Deformable Porous Media}

In this section we derive the equations describing multiphase, multicomponent thermal flow in deformable porous media. The derivation is motivated by the formulation used in Martinez et al. (2001) for rigid porous media and by the formulation in Rutqvist et al. (2001).

\section{Balance Equations for a Phase}

The general macroscopic balance equation for the mass or energy density of a component $\alpha$ in phase $\beta$ can be written as

$$
\frac{\partial d_{\beta}^{\alpha}}{\partial t}+\nabla \bullet \mathbf{q}_{\beta}^{\alpha}-Q_{\beta}^{\alpha}=0
$$

where $d$ is the mass or energy density, q is the flux density with respect to a reference coordinate system and $Q$ is a source term. For example, a typical system might consider water, air and energy (as the components) distributed over a liquid and gas phase. The mass density is

$$
d_{\beta}^{\alpha}=\phi S_{\beta} \rho_{\beta}^{\alpha}
$$

in which $\phi$ denotes the porosity, $S_{\beta}$ the phase saturation and $\rho$ is the mass density of component $\alpha$ per unit volume of phase $\beta$. In anticipation of solid motion in a deformable porous medium, the flux density is written as

$$
\mathbf{q}_{\beta}^{\alpha}=\mathbf{q}_{r, \beta}^{\alpha}+\phi S_{\beta} \rho_{\beta}^{\alpha} \mathbf{v}_{s}
$$

where $\mathbf{q}_{r, \beta}^{\alpha}$ is the flux density relative to the moving solid, and $\mathbf{v}_{s}$ is the solid velocity. The relative flux density is written as,

$$
\mathbf{q}_{r, \beta}^{\alpha}=\phi S_{\beta} \rho_{\beta}^{\alpha} \mathbf{v}_{r, \beta}+\mathbf{J}_{\beta}^{\alpha}
$$

where the relative phase velocity is,

$$
\mathbf{v}_{r, \beta}=\mathbf{v}_{\beta}-\mathbf{v}_{s},
$$

and $\mathbf{J}_{\beta}^{\alpha}$ is the diffusive/dispersive mass flux. Notice that the foregoing relations are written with respect to "pore velocities," as opposed to Darcy velocities. Darcy velocity is the mass flux per unit area of porous material, whereas pore velocity is the average fluid velocity in the pores of the material. Darcy velocity ( $\mathbf{V})$ is related to pore velocity according to

$$
\mathbf{V}_{\beta}=\phi S_{\beta} \mathbf{v}_{\beta} .
$$

Now, if we substitute the expressions for density and flux into the general balance equation, we get

$$
\frac{\partial}{\partial t}\left(\phi S_{\beta} \rho_{\beta}^{\alpha}\right)+\nabla \bullet\left(\phi S_{\beta} \rho_{\beta}^{\alpha} \mathbf{v}_{\beta}+\mathbf{J}_{\beta}^{\alpha}\right)+Q_{\beta}^{\alpha}=0 .
$$

If we apply this to the solid phase (unit phase saturation, no diffusive components, no sources) we get the balance law for the porous solid,

$$
\frac{\partial}{\partial t}\left((1-\phi) \rho_{s}\right)+\nabla \bullet\left((1-\phi) \rho_{s} \mathbf{v}_{s}\right)=0
$$


In order to develop the contributions to the balance equation from a deformable matrix, we introduce the relative and solid velocity in eqn. (10) and expand the resulting flux term to arrive at the Lagrangian form of the general balance equation,

$$
\frac{D}{D t}\left(\phi S_{\beta} \rho_{\beta}^{\alpha}\right)+\nabla \bullet \mathbf{q}_{r, \beta}^{\alpha}+\phi S_{\beta} \rho_{\beta}^{\alpha} \nabla \bullet \mathbf{v}_{s}+Q_{\beta}^{\alpha}=0,
$$

where we have introduced the material derivative following the motion of the solid,

$$
\frac{D}{D t}=\frac{\partial}{\partial t}+\mathbf{v}_{s} \bullet \nabla
$$

This form of the balance equation explicitly includes the effect of deformation in the divergence of solid phase velocity. In order to develop a rate equation for the evolution of porosity, we expand eqn. (11) and make use of the material derivative to get

$$
\frac{D \phi}{D t}=\frac{1-\phi}{\rho_{s}} \frac{D \rho_{s}}{D t}+(1-\phi) \nabla \bullet \mathbf{v}_{s} .
$$

If we expand the material derivative term in eqn. (10) and make use of the evolution equation for porosity we get an intermediate Lagrangian balance equation,

$$
\phi \frac{D}{D t}\left(S_{\beta} \rho_{\beta}^{\alpha}\right)+\nabla \bullet \mathbf{q}_{r, \beta}^{\alpha}+S_{\beta} \rho_{\beta}^{\alpha}\left(\nabla \bullet \mathbf{v}_{s}+\frac{(1-\phi)}{\rho_{s}} \frac{D \rho_{s}}{D t}\right)+Q_{\beta}^{\alpha}=0 .
$$

To simplify the formulation further, we resort to invoking several small strain approximations, also made by others (Rutqvist et al, Lewis and Schrefler). These approximations include,

1. $\nabla \bullet \mathbf{v}_{s}=\frac{D \varepsilon_{V}}{D t} \approx \frac{\partial \varepsilon_{V}}{\partial t} \quad \nabla \varepsilon_{V} \ll \partial \varepsilon_{V} / \partial t$

2. $\mathbf{v}_{s} \bullet \nabla\left(S_{\beta} \rho_{\beta}^{\alpha}\right) \ll \partial\left(S_{\beta} \rho_{\beta}^{\alpha}\right) / \partial t$

3. $\mathbf{v}_{s} \bullet \nabla \rho_{s} \ll \partial \rho_{s} / \partial t$

In the first of these we have introduced the volume strain $\varepsilon_{V}=\nabla \cdot \mathbf{u}$ in terms of the divergence of the displacement vector, (u). Applying these approximations results in the working version of the balance equation for component $\alpha$ in phase $\beta$, including the effects of deformation,

$$
\phi \frac{\partial}{\partial t}\left(S_{\beta} \rho_{\beta}^{\alpha}\right)+\nabla \bullet \mathbf{q}_{r, \beta}^{\alpha}+S_{\beta} \rho_{\beta}^{\alpha}\left(\frac{\partial \varepsilon_{V}}{\partial t}+\frac{(1-\phi)}{\rho_{s}} \frac{\partial \rho_{s}}{\partial t}\right)+Q_{\beta}^{\alpha}=0 .
$$

Before applying this equation for the multiphase multicomponent system, we introduce some convenient alternative formulations. Computational experience with multiphase systems has shown that mass conservation in time-dependent problems is better modeled when the porosity is included inside the time derivative. If we simply use the chain rule on the time term we get

$$
\frac{\partial}{\partial t}\left(\phi S_{\beta} \rho_{\beta}^{\alpha}\right)+\nabla \bullet \mathbf{q}_{r, \beta}^{\alpha}+S_{\beta} \rho_{\beta}^{\alpha}\left(\frac{\partial\left(\varepsilon_{V}-\phi\right)}{\partial t}+\frac{(1-\phi)}{\rho_{s}} \frac{\partial \rho_{s}}{\partial t}\right)+Q_{\beta}^{\alpha}=0 .
$$

We introduce a further manipulation to derive an approximate expression for the evolution of porosity, invoking the "incompressible solid" approximation. This amounts to assuming

$$
\frac{1-\phi}{\rho_{s}} \frac{D \rho_{s}}{D t} \ll(1-\phi) \nabla \bullet \mathbf{v}_{s} \text {, }
$$


therefore the evolution equation for porosity in eqn. (14) is approximated by,

$$
\frac{1}{1-\phi} \frac{D \phi}{D t}=\frac{D \varepsilon_{V}}{D t} \text {. }
$$

If we integrate this from a state of zero strain to finite strain, we get (e.g. Kowalski \& Rybicki, 2004)

$$
\ln \frac{1-\phi_{0}}{1-\phi}=\varepsilon_{V}=\nabla \bullet \mathbf{u} .
$$

If we make use of this (or in the form of eqn. (17)), in the foregoing form of the balance equation, we get the alternative form,

$$
\frac{\partial}{\partial t}\left(\phi S_{\beta} \rho_{\beta}^{\alpha}\right)+\nabla \bullet \mathbf{q}_{r, \beta}^{\alpha}+S_{\beta} \rho_{\beta}^{\alpha}\left(\phi \frac{\partial \varepsilon_{V}}{\partial t}+\frac{(1-\phi)}{\rho_{s}} \frac{\partial \rho_{s}}{\partial t}\right)+Q_{\beta}^{\alpha}=0
$$

For a rigid porous medium, the terms involving solid dilatation and grain compressibility are absent.

\section{Balance Equation for Mass Components}

For multiphase systems with phase transitions (e.g. evaporation, condensation) of mass components, it is convenient to work with mass balance equations for components. These are derived by summing the general phase balance equation over all fluid phases. In this procedure, the part of the source terms that relate to mass transfer between phases cancels out, assuming equilibrium. This can be written as,

$$
\frac{\partial}{\partial t}\left(\sum_{\beta} \phi S_{\beta} \rho_{\beta}^{\alpha}\right)+\nabla \bullet \sum_{\beta} \mathbf{q}_{r, \beta}^{\alpha}+\left(\sum_{\beta} S_{\beta} \rho_{\beta}^{\alpha}\right)\left(\phi \frac{\partial \varepsilon_{V}}{\partial t}+\frac{(1-\phi)}{\rho_{s}} \frac{\partial \rho_{s}}{\partial t}\right)+\sum_{\beta} Q_{\beta}^{\alpha}=0
$$

As a concrete example, a typical two-phase system is a mixture of water and air in liquid and gas phases. The total mass balance for water is,

$$
\frac{\partial}{\partial t}\left(\phi\left(S_{l} \rho_{l}^{w}+S_{g} \rho_{g}^{w}\right)\right)+\nabla \bullet\left(\mathbf{q}_{r, l}^{w}+\mathbf{q}_{r, g}^{w}\right)+\left(S_{l} \rho_{l}^{w}+S_{g} \rho_{g}^{w}\right)\left(\phi \frac{\partial \varepsilon_{V}}{\partial t}+\frac{(1-\phi)}{\rho_{s}} \frac{\partial \rho_{s}}{\partial t}\right)+Q^{w}=0
$$

and for the air

$$
\frac{\partial}{\partial t}\left(\phi\left(S_{l} \rho_{l}^{a}+S_{g} \rho_{g}^{a}\right)\right)+\nabla \bullet\left(\mathbf{q}_{r, l}^{a}+\mathbf{q}_{r, g}^{a}\right)+\left(S_{l} \rho_{l}^{a}+S_{g} \rho_{g}^{a}\right)\left(\phi \frac{\partial \varepsilon_{V}}{\partial t}+\frac{(1-\phi)}{\rho_{s}} \frac{\partial \rho_{s}}{\partial t}\right)+Q^{a}=0
$$

\section{Heat Transport}

The literature is not as complete on derivations of the energy equation. Lewis and Schrefler ultimately advocate a thermal energy equation with an explicit latent heat term to account for evaporation/condensation, see their eqn. (2.334). This is a cumbersome method for treating phase change. Instead we follow the formulation in Martinez et al. (2001), which is derived for rigid porous media:

$$
\frac{\partial}{\partial t}\left[(1-\phi) \rho_{s} e_{s}+\phi\left(\rho_{l} S_{l} e_{l}+\rho_{g} S_{g} e_{g}\right)\right]+\nabla \bullet \mathbf{q}_{e}=Q_{e}
$$


The total heat flux vector includes heat conduction, convection (modified for deformation), and heat transport due to binary diffusion,

$$
\mathbf{q}_{e}=-\lambda_{T} \nabla T+\sum_{\beta} \mathbf{q}_{r, \beta} h_{\beta}+\sum_{\alpha} h_{g}^{\alpha} \mathbf{J}_{g}^{\alpha}
$$

where the diffusive fluxes are approximated as,

$$
\mathbf{J}_{g}^{\alpha}=-\rho_{g} D_{g}^{\alpha} \nabla Y_{g}^{\alpha}
$$

and $Y_{g}^{\alpha}$ is the mass fraction of component $\alpha$ in the gas phase,

$$
Y_{g}^{\alpha}=\frac{\rho_{g}^{\alpha}}{\rho_{g}} .
$$

\section{Summary}

The foregoing formulation forms the basis for the flow and energy equations describing multiphase multicomponent thermal transport in deformable porous media. In particular the initial implementation would most likely constitute the air and water balance equations (21) and (22), plus the heat balance in (23). The quasi-static deformation mechanics would be described by the linear momentum equation (2), ignoring acceleration and damping (the RHS) and utilizing the effective stress principle in a form similar to equation (1). 


\section{PHASE TRANSFORMATIONS}

Specialized algorithmic approaches are necessary for the numerical solution of problems involving multiphase multicomponent flow in which phases may appear and disappear (e.g. evaporating or condensing flow going from two phase to single phase or vice versa). A phase transition is manifested as a discontinuous trace in a thermodynamic surface. Numerical instabilities will likely occur whenever there is a phase appearance or disappearance regardless of the choice of independent variables because the Jacobian is discontinuous at that point. This section summarizes our research into the literature on numerical methods to deal with phase appearance and disappearance in multiphase multicomponent flows in porous materials.

\section{Methods Investigated}

In reviewing the literature, the following methods and techniques appear to be most relevant to the current project:

- Pseudo-variables

- Relaxed volume balance

- Mixture model (Wang et al., 1996)

- Persistent variables

- Variable switching

Following are notes on some of these methods. Other methods were not considered at the outset due to certain undesirable properties. The pseudo-variables method, as discussed for example by Kirkland et al. (1992), and Binning \& Celia (1999), appears unwieldy and doesn’t seem generally applicable to multiphase, multicomponent problems. Basically, working with a pseudovariable is unwieldy when trying to apply BCs and in the presence of formation heterogeneity.

\section{Relaxed Volume Balance}

This is not exactly a method for dealing with phase transitions, but it may help in dealing with volume and mass balance issues in these problems. Acs et al. (1985) appear to have devised this method. They formulate a global pressure equation from what is essentially a constraint equation stating that the fluid volume must occupy the porous volume in the formation. The final pressure equation couples all component masses, fluxes and partial volumes, and is always valid independent of the phase configuration. Coats et al. (1998) indicate the method reduced the iteration counts compared to other methods in which the auxiliary constraints are included in the equations sets. The model discussed in Coats et al. (1998) starts with the constraint equations (consisting of the saturation and phase mass/mole fractions summing to unity, and the phase equilibrium constraints, deriving from equal chemical potential in each coexisting phase for each component) coupled in with the conservation equations in one global matrix. The constraint equations are condensed out prior to solving the main system of nonlinear algebraic equations. The terms in the equations are constructed so that the mass fluxes are in balance exactly (numerically). The essence of the relaxed volume concept is that the saturation constraint is not applied in setting up the main system, but instead is used as a convergence constraint on volume. 
Coats (2000) discusses a general way of generating the IMPES (implicit pressure explicit saturation) pressure equation, which seems related to the issue of the relaxed volume concept, the fractional flow formulation and Wang's mixture model, to be discussed shortly. IMPES is one of the classic methods of solving the reservoir modeling problem. Basically, the pressure equation is derived from the (assembled) nodal equations at a grid point. He describes a general method of elimination, which involves solving for an "IMPES reduction vector," to calculate a pressure equation which is a linear combination of the $n$ component equations at a grid block. Coats implies that the resulting pressure equation is easier (smoother, etc.) to solve for compared to solving all equations simultaneously. In addition, as the acronym implies, the saturation equations are then solved explicitly. This may be advantageous when the capillary effects are subdominant to other driving forces in the problem.

An interesting feature of Coat's paper is the way he writes his Newton iteration equations, see his eqns. (2) and (3a). Eqn. (2) is just a rewriting of the governing equations, eqn. (1), in terms of the iteration level $l+1$, arranged into what some authors call a "delta" form. That is, write eqn. (1) at iteration level $l+1$, and add and subtract the storage and flux operator terms at level $l$, then re-arrange to get eqn. (2). Notice that to get his Newton system, eqn. (3a), he apparently applies Taylor approximation to the time term only,

$$
\delta M_{i}=M_{i}^{l+1}-M_{i}^{l}=\frac{\partial M_{i}}{\partial P_{j}} \delta P_{j}=g_{i j}\left(P_{j}^{l+1}-P_{j}^{l}\right),
$$

and leaves the fluxes in the delta form without a Jacobian contribution. Coats does not elaborate on whether this form of linearization is better than the standard full Newton scheme, which would have Jacobian contributions for all the terms in the equation.

\section{Wang's Mixture Model}

In a series of papers Wang and co-workers (Wang \& Cheng, 1996; Cheng \& Wang, 1996) introduce a scheme that involves a system mixture pressure (pseudo-pressure) which is persisent, that is, independent of the existing phase state. They arrange the multiphase, multicomponent flow equations into a mixture formulation, in which the dependent variables are mixture values of density, Darcy velocity, pressure, etc. Contrary to their claims, this method does not reduce the number of PDEs that need be solved in these types of problems. Some of the issues related to this formulation follow.

In the two-phase two-component model, they solve for a mixture pressure and air concentration. The mixture pressure is solved from the mixture mass balance equation, which is analogous to the IMPES scheme, (except the equations are solved simultaneously), or what is called the fraction flow approach, (e.g. Binning \& Celia, 1999).

Incidentally, Binning \& Celia (1999) cite problems with the mixture pressure approach when applying general boundary conditions, mainly because of the pseudo-pressure used in the model. The pseudo-pressure is the variable solved for from the "pressure" equation, which is analogous to Wang's mixture pressure and mixture mass balance equation. They cite as an example a problem where one wants to specify air pressure and water flux at an "atmospheric" boundary. One cannot compute directly the mixture pressure from these two physical quantities, as needed 
for a boundary condition on the pressure equation. Binning \& Celia solve this by developing an iterative procedure to compute the mixture variables that would enforce the desired boundary quantities. They also note that a different iterative algorithm is needed for each combination of flux and saturation boundary condition pair. A similar issue would seem to arise in Wang's formulation when one wants to apply a flux for some components and pressures (or mass fractions) for others at a boundary. One would have to devise a way of computing the mixture pressure from a mixture of compositions and fluxes.

The resulting equations in Wang's formulation are nonlinear, although without experience it is difficult to assess how difficult they are to solve. Wang appears to solve them coupled, but segregated, though it is not entirely clear from this discussion. (Binning \& Celia, with a similar formulation, say their pressure equation is well-behaved and nicely elliptic.) The concentration equation is solved using some form of upwinding for convective fluxes.

The several conclusions in Binning \& Celia with regard to the fractional flow formulation would seem to apply to Wang's similar mixture formulation. There are difficulties with general boundary conditions, heterogeneous materials, and mass conservative schemes when using methods of characteristic based methods of solution of the hyperbolic concentration equations. It appears that there are too many outstanding issues with the mixture formulation to risk development of a general purpose simulator based on this formulation.

\section{Persistent Variables}

An obvious approach to dealing with phase appearance and disappearance is to select a set of persistent solution variables, meaning variables which exist for any of the possible phase configurations. However, such a set excludes certain variables known to yield favorable numerical algorithms under certain phase states. An example is phase saturation; liquid or gas saturation is a common and favorable choice in a gas/liquid system. However, phase saturation ceases to be a "variable" when the system transitions to single phase (gas or liquid), where it is a constant.

Corapcioglu \& Panday (1991) and Panday et al. (1995) discuss a “Z formulation,” which is a persistent variables approach. The $\mathrm{Z}$ variable(s) represents the total mole fraction of a component in the multiphase mixture. Actually, their formulation would solve for a pressure and the remainder of $\mathrm{Z}$ values for the other components. For a general compositional model, flash calculations are needed to compute compositions from the Z-values. (See Lake, 1989, Enhanced Oil Recovery, for an example of a flash calculation) For simpler systems, like problems involving non-aqueous phase liquids (NAPLs), the flash calculations can often be avoided with judicious choices of primary variables. This was the choice of method that was finally implemented in Panday et al (1995). They argue that the most promising method with respect to efficiency is a combination of the full $\mathrm{Z}$ formulation and a variable switching scheme.

A persistent variables scheme is used in PorSalsa (Martinez et al., 2001), an SNL nonisothermal two-phase flow code. The independent variables include total (both liquid and gas phase) water density, which is analogous to the total mole fraction or $\mathrm{Z}$ variable of Corapcioglu \& Panday (1991). The performance of PorSalsa with respect to phase transitions is problem dependent. 
Persistent variables are used in the ACRES oil reservoir simulator (R. Dean, Chevron, personal communication), which solves both thermal and isothermal compositional problems.

In a recent paper Krabbenhoft (2007) discusses an alternative to variable switching for treating phase appearance and disappearance, though only in the context of saturated-unsaturated flow (the method is not directly applicable to the transition from two-phase to all-vapor). The technique utilizes the standard pressure formulation, though the flow equations are developed in the mixed formulation (with both saturation and pressure appearing), with saturations computed from the retention curve knowing the grid values of pressure. In a sense, the method transfers the variable switching to the update stage in the Newton iteration cycle. During Newton iteration, a pressure update is computed as usual. However, in the update stage, the algorithm computes all the implied "provisional" updated saturations, from the retention curve. The algorithm utilizes tolerances to determine whether to apply the pressure update as usual, or whether to accept the provisional saturation and compute the updated pressure using this saturation in the retention curve. Krabbenhoft reports good performance, and notes the ease with which this technique can be implemented in existing pressure based formulations.

\section{Variable Switching}

Variable switching is a specialized algorithmic approach to the numerical solution of problems involving multiphase multicomponent flow in which phases may appear and disappear. A phase transition appears as a discontinuous trace in a thermodynamic surface. Nonlinear solution schemes such as Newton iteration assume smooth functions. The switching of solution variables upon phase appearance or disappearance has the potential to tame the nonlinearities associated with numerical solutions of systems of equations with discontinuous variables. The idea is to substitute the (unknown) solution variable from one that is discontinuous (or nonexistent in the new phase configuration) across the phase transition to one that is smooth in the new phase configuration. The method thus requires a phase diagnosis algorithm to detect phase appearances or disappearances and a scheme to initialize the new variable, which may or may not have existed in the previous phase configuration.

Early practitioners of the variable substitution scheme include Coats (1980a,b) and Fussell and Fussell (1979), applied to compositional reservoir simulation. A more recent paper by Panday et al (1995) applies a "composite” technique, combining the persistent variable " $Z$ ” scheme discussed earlier with a variable switching scheme, patterned after Forsyth \& Shao (1991). The Z scheme apparently requires some flash calculations, which impairs its numerical efficiency. Recall that the $\mathrm{Z}$ variables represent the mass or mole fraction of a component in the porous structure, summed over all the various existing phases. As such, it is not surprising that a flash calculation would be required to extract required intensive variables, like pressure and temperature. On the other hand, the variable switching scheme of Forsyth \& Shao was formulated to switch out all the phase mass fractions from a disappearing phase to those of another existing phase. Also, re-initialization criteria for switched variables are not entirely transparent in this scheme when air is present. The method studied and implemented by Panday et al. is a combination of appealing aspects of these two methods. The main features of the combined method include: 
- Provides variable switching criteria when air is present - which was apparently not the case in the method of Forsyth \& Shao.

- Using a judicious choice of primary variables, including some Z variables, pressure, and temperature, fewer variables need to be switched (and re-initialized) upon a phase appearance/disappearance.

- $\quad$ Rather than the more complex (and nonlinear) flash required in the $\mathrm{Z}$ formulation, only a mildly nonlinear problem is required for computing secondary variables from primary variables.

- The authors state that the matrix is more diagonally stable in the presence of noncondensible gases.

After testing on several problems, they find:

- The present model is more robust on isothermal problems. The new model was at least as robust as recent models (Forsyth \& Shao, 1991) on nonisothermal problems.

- This model is more general, not requiring infinitesimal amounts of certain phases/components as is needed in the other models.

- Variable switching tends to "jolt" the system and requires more Newton iterations. The issue, they believe, is that upon switching, the re-initialized values of the switched variables are not consistent with the new state. They tried predictor methods, but observed mixed results.

- They also had problems with a "penalty source" used in nonisothermal problems when inert components are present (e.g., air). The source introduces masses which have to be iterated out by the Newton scheme - resulting in a source of inefficiency.

In a two paper series, Class et al. (2002) and Class \& Helmig (2002) discuss the use of variable switching in a 3-phase, 3-component thermal model. This represents an extension of the work of Forsth \& Shao (1991). Class \& Helmig show an application (1D) with steam displacing/distilling out a NAPL completely $\left(S_{\mathrm{n}}=0\right)$ over part of the domain. Other notables from this work:

- Class \& Helmig, in Figure 4, discuss a phase diagram for two components of limited mutual miscibility. They ultimately simplified the numerical implementation using Dalton's law to sum up vapor pressures for components to make up the gas pressure.

- $\quad$ They give criteria for phase diagnosis, Table 2 in Class et al. (2002).

- $\quad$ They note problems with the variable switching scheme in the case of phase appearance.

Diersch \& Perrochet (1999) also studied the use of variable switching in saturated/unsaturated flow. Like Forsyth et al. (1995), they suggest that these "mixed" schemes are very effective for this class of problem, especially for dry initial conditions and for saturated/unsaturated transitions. 


\section{Recommendation}

Unfortunately, it is not possible to determine the best technique from the short literature review performed. Improved performance with variable switching is reported for isothermal saturated/unsaturated problems, but mixed results are reported for nonisothermal multiphase problems. Mixed results have also been reported using persistent variables, though this technique is used in some reservoir simulators.

In the interest of developing a leading-edge multiphase multicomponent simulation capability, it may be best to prototype some of the techniques discussed above. A flexible architecture is the key piece that would enable the prototyping. An architecture that enables persistent variable or variable switching is needed. This could be accomplished by coding all residual equation assembly, formation and transport properties (i.e., secondary variables) routines so that they may be invoked as one or a few number of functions. Similarly, the thermodynamic equation of state and other PVT routines should be callable as functions. Then a forward difference approximation to the Jacobian matrix in a Newton iteration scheme can be assembled easily. For variable switching, the variable substitution can occur within the Newton iteration loop, and otherwise requires the thermodynamic function to be able to return secondary variables based on the currently existing phase state. A variable initialization function is also required to reset values for switched variables. 


\section{DEVELOPMENT PLAN}

The ultimate goal of the proposed development project is a state-of-the-art, extensible, simulation capability and platform to address multiphase, multicomponent thermal flow and reactive transport coupled to nonlinear geomechanics in heterogeneous (geologic) porous materials. A plan for the future development of these models is presented in this section. We intend to enable the development of this new multiphysics capability by a seamless, flexible coupling of individual physics modules utilizing the SNL Sierra platform. Sierra would enable this project by providing unique leading-edge software tools necessary to achieve efficient, stable and robust coupling algorithms for the aforementioned physics modules.

The key R\&D goals include:

- Development of a coupled multiphysics solution package for geosystems management, including thermal, hydrological, mechanical and chemistry for subsurface problems

- Development of adaptive solution controls to enable performance efficiency of the coupled multiphysics simulator

A suggested task plan to accomplish the development goals are as follows:

Year 1:

- Develop and verify geomechanics-specific capabilities in Sierra/Adagio

- Develop and verify nonisothermal multiphase, multicomponent (air/water) capability in Sierra Mechanics

- Identify and select geochemistry package for use in coupled reactive transport

- Implement an initial adaptive control strategy for coupled flow and geomechanics

- Solve a coupled saturated flow problem with geomechanics

Year 2:

- Develop a multiphase equation-of-state based compositional capability (applicable to $\mathrm{CO}_{2}$ sequestration)

- Solve a coupled multiphase flow problem with geomechanics

- Implement adaptive control algorithm for flow and geomechanics

- Develop initial reactive transport module

- Develop coupling of reactive (geochemistry) transport with multiphase flow Year 3:

- Complete compositional flow capability development

- Complete reactive transport module

- Resolve any remaining solver issues between multiphysics couplings

- Begin verification phase of the coupled system and subsystems and compare to available validation-quality data

- Refine adaptive control algorithms based on performance on benchmark tests 


\section{REFERENCES}

Acs G, Doleschall S, and Farkas E. (1985) General purpose compositional model, Society of Petroleum Engineers Journal, 25 (4), 543-553.

Alonso EE, Gens A, Josa A. (1990) A constitutive model for partially saturated soils, Geotechnique 40, 405-430.

Binning P and Celia M. (1999) Practical implementation of the fractional flow approach to multi-phase flow simulation, Advances in Water Resources, 22 (5), 461-478.

Biot, MA. (1941) General theory of three dimensional consolidation, Journal of Applied Physics, 12, $155-164$.

Biot MA and Willis PG. (1957) The elastic coefficients of the theory of consolidation, Journal of Applied Mechanics, 24, 594-601.

Cheng P and Wang CY. (1996) A multiphase mixture model for multiphase, multicomponent transport in capillary porous media - II. Numerical simulation of the transport of organic compounds in the subsurface, International Journal of Heat and Mass Transfer, 39 (17), pp.3619-3632.

Class H, Helmig R and Bastian P. (2002) Numerical simulation of non-isothermal multiphase multicomponent processes in porous media. 1. An efficient solution technique, Advances in Water Resources, 25, 533-550.

Class H and Helmig R. (2002) Numerical simulation of non-isothermal multiphase multicomponent processes in porous media. 2. Applications for the injection of steam and air, Advances in Water Resources, 25, 551-564.

Coats KH. (2000) A note on IMPES and some IMPES-based simulation methods, SPE Journal 5 (3), (September), 245-251.

Coats KH, Thomas LK, and Pierson RG. (1998) Compositional and black oil reservoir simulation, SPE Reservoir Evaluation \& Engineering, (August), 372-379.

Corapcioglu MY and Panday S. (1991) Compositional multiphase flow models, Advances in Porous Media, 1, 1-59.

Diersch H-JG and Perrochet P. (1999) On the primary variable switching technique for simulating unsaturated-saturated flows, Advances in Water Resources, 23, 271-301.

De Boer R. (1998)Theory of porous media - past and present, Z. Angew. Math. Mech., 78 (7), 441-466.

Dean RH, Gai X, Stone CM, and Minkoff SE. (2003)A comparison of techniques for coupling porous flow and geomechanics, SPE 79709, SPE Reservoir Simulation Symposium, Houston Texas, February 3-5.

Forsth PA and Shao BY. (1991) Numerical simulation of gas venting for NAPL site remediation, Advances in Water Resources, 14, 354-367. 
Forsyth PA, Wu YS, and Pruess K. (1995) Robust numerical methods for saturated-unsaturated flow with dry initial conditions in heterogeneous media, Advances in Water Resources, 18, 25-38.

Kirkland MR, Hills RG, and Wierenga PJ. (1992) Algorithms for solving Richards equation for variably saturated soils, Water Resources Research, 28 (8) 2049-2058.

Krabbenhoft K. (2007) An alternative to primary variable switching in saturated-unsaturated flow computations, Advances in Water Resources, 30, 483-492.

Kowalski SJ, and Rybicki A. (2004) Drying induced stresses in a swelling porous wall, Transport in Porous Media, 57, 35-48.

Lake LW. (1989) Enhanced Oil Recovery, Prentice-Hall Inc., Englewood Cliffs, New Jersey.

Lewis RW and Schrefler BA. (1998) The Finite Element Method in the Static and Dynamic Deformation and Consolidation of Porous Media, $2^{\text {nd }}$ Ed., Wiley, New York.

Lo W-C, Esposito G, and Majer E. (2002) Immiscible two-phase flows in deformable porous media, Advances in Water Resources, 25, 1105-1117.

Martinez MJ, Hopkins PL, and Reeves PC. (2001) PorSalsa User's Manual, Sandia National Laboratories Tech. Rept, SAND2001-1555, Albuquerque, NM.

Panday S, Forsyth PA, Falta RW, Wu Y-S, and Huyakorn PS (1995) Considerations for robust compositional simulations of subsurface nonaqueos phase liquid contamination and remediation, Water Resources Research, 31 (5), 1273-1289.

Rutqvist J, Borgesson L, Chijimatsu M, Kobayashi A, Jing L, Nguyen TS, Noorishad J, and Tsang C-F. (2001) Thermohydromechanics of partially saturated geologic media: governing equations and formulation of four finite element models, International Journal of Rock Mechanics \& Mining Science, 38, 105-127.

Rutqvist J, Wu Y-S, Tsang C-F, and Bodvarsson G. (2002) A modeling approach for analysis of coupled multiphase fluid flow, heat transfer, and deformation in fractured porous rock, International Journal of Rock Mechanics \& Mining Science, 39, 429-442.

Schrefler BA and Scotta R. (2001) A fully coupled dynamic model for two-phase fluid flow in deformable porous media, Computational Methods in Applied Mechanics and Engineering, 190, 3223-3246.

von Terzaghi, K. (1943) Theoretical Soil Mechanics, Wiley, New York.

Wang CY and Cheng P. (1996) A multiphase mixture model for multiphase, multicomponent transport in capillary porous media - II. Model development, International Journal of Heat and Mass Transfer, 39 (17), 3607-3618. 


\section{Distribution:}

$\begin{array}{llll}1 & \text { MS0157 } & \text { H. S. Morgan } & 1030 \\ 1 & \text { MS0372 } & \text { C. M. Stone } & 1525 \\ 1 & \text { MS0382 } & \text { H. C. Edwards } & 1541 \\ 1 & \text { MS0382 } & \text { P. K. Notz } & 1541 \\ 1 & \text { MS0751 } & \text { T. Dewers } & 6315 \\ 5 & \text { MS0836 } & \text { M. J. Martinez } & 1514 \\ 1 & \text { MS1318 } & \text { R. Hooper } & 1414 \\ & & & \\ 1 & \text { MS0899 } & \text { Technical Library } & 9536 \\ 1 & \text { MS0123 } & \text { D. Chavez, LDRD Office } & 1011\end{array}$




\section{(1) Sandia National Laboratories}

